\title{
Adaptación del Cuestionario para Identificación de Trastornos Debidos al Consumo de Alcohol (AUDIT) en universitarios colombianos
}

\author{
Fernando Riveros ${ }^{1}$, Luis A. Vera ${ }^{2}$, Carlos Gantiva ${ }^{2}$ y Leidy Torres ${ }^{3}$ \\ ${ }^{1}$ Universidad de La Sabana, Chía, Colombia \\ ${ }^{2}$ Universidad de San Buenaventura, Bogotá, Colombia \\ ${ }^{3}$ Corporación Universitaria Iberoamericana, Bogotá, Colombia
}

\begin{abstract}
Adaptation of the Alcohol Use Disorders Identification Test (AUDIT) in Colombian university students. Alcohol consumption is one of the behaviors that generate more negative consequences for public health, but there are few validated instruments for measuring alcohol consumption in the Colombian population. The objective of the present investigation was to adapt the Alcohol Use Disorders Identification Test (AUDIT) for use with Colombian university students. The method used was instrumental, because the questionnaire was submitted to the technical adaptation process defined by WHO. It was applied to a sample of 1,557 Colombian university students, selected with a probabilistic procedure. The results showed a scale with adequate clarity of language with two clearly identified components that explain $38.54 \%$ of the accumulated variance, a model that was confirmed and Cronbach's alpha of .82 for the total scale, being .73 for the first component and .79 for the second component and with positive correlations between the items. The AUDIT Test is an adapted scale with appropriate psychometric performance for use with Colombian university students.
\end{abstract}

Keywords: Alcohol consumption; AUDIT; assessment.

Resumen: El consumo de alcohol es una de las conductas que genera mayores problemáticas para la salud pública, pero son escasos los instrumentos validados que permiten medirlo en población colombiana. El objetivo de la presente investigación fue adaptar el Cuestionario para Identificación de Trastornos Debidos al Consumo de Alcohol (AUDIT) en universitarios colombianos. El método utilizado fue instrumental, dado que el cuestionario fue sometido al proceso técnico de adaptación definido por la OMS. Se aplicó a una muestra de 1.557 universitarios colombianos, seleccionados bajo un procedimiento probabilístico. Los resultados mostraron que la escala (a) posee claridad adecuada en su lenguaje, (b) se ajusta a un modelo de dos factores bien definidos, y (c) posee buenos niveles de fiabilidad (consistencia interna) y correlaciones positivas entre los reactivos. Se concluye que el AUDIT es un instrumento con propiedades psicométricas apropiadas para ser utilizado en universitarios colombianos.

Palabras clave: Consumo de alcohol; AUDIT; evaluación.

\section{Introducción}

El consumo excesivo de alcohol es un problema de salud pública a nivel nacional e internacional. Los datos indican no solo un efecto dañino sino también una alta prevalencia de dicho consumo problémico. En torno a las consecuencias del consumo, por resaltar algunas, se en-

Recibido: 26 de junio de 2018; aceptado: 12 de septiembre de 2018 Correspondencia: Fernando Riveros Munévar, Universidad de La Sabana, Km. 7, Autopista Norte de Bogotá, Chía, Colombia. Correo-e: efriveros45@hotmail.com cuentran intoxicaciones en algunos casos fatales, afectaciones en ámbitos académicos y laborales, altercados en las relaciones interpersonales, conductas de riesgo a nivel sexual, accidentes de tráfico, riñas, lesiones, entre otras (Motos, Cortés, Giménez, y Cadaveira, 2015), consumo junto a otras sustancias psicoactivas de mayor impacto, trastornos del estado de ánimo, y trastornos de personalidad antisocial y límite (Grant et al., 2015).

Según el último informe de la Organización Mundial de la Salud (OMS, 2014), el consumo de alcohol en personas mayores de 15 años es de 6.2 litros de alcohol puro por año, lo que equivale a un consumo diario de 13.5 
gramos de alcohol puro. Del total de alcohol consumido a nivel mundial, el $24.8 \%$ es elaborado de forma artesanal, sin ningún tipo de control estatal o sanitario y el $50.1 \%$ proviene de bebidas destiladas, las cuales tienen la mayor concentración de alcohol puro. Se estima que el $16 \%$ de los consumidores de alcohol mayores de 15 años tienen episodios de consumo excesivo de alcohol. Las regiones con mayores índices de consumo de alcohol per capita son Europa y América, en general se observa que a mayor es el desarrollo económico de un país y su riqueza, mayor es el nivel de consumo de la población y menor el índice de personas abstinentes, de hecho, se observa como regla que los países con mayores ingresos tienen los mayores índices de consumo de alcohol per capita y los mayores índices de prevalencia de consumo excesivo (OMS, 2014). En ese sentido, los datos estadounidenses refieren prevalencias de trastornos del consumo de alcohol en el ultimo año en el 13.9\% y en la vida en el 29.1\% de la población adulta (Grant et al., 2015).

A nivel latinoamericano, el estudio de Cremonte, Biscarra, Conde y Cherpitel (2018) refiere que el consumo de alcohol es la principal causa de muerte entre los hombres de 15 a 49 años de edad y la tercera en mujeres de la región andina; sin embargo, los autores también resaltan la escasez de estudios de corte epidemiológico al respecto. La bebida alcohólica más consumida en Colombia es la cerveza (66\%), seguida por las bebidas destiladas (33\%). Se estima que la prevalencia de episodios de consumo excesivo en hombres mayores de 15 años consumidores de alcohol es de $13.8 \%$, mientras que para las mujeres es de $0.6 \%$. Con relación a las consecuencias, se estima que el $56 \%$ de las cirrosis en hombres y el $54.2 \%$ de la cirrosis en mujeres se deben al consumo excesivo de alcohol y que el $23.2 \%$ de los accidentes de tránsito ocasionados por hombres y el $9 \%$ de los ocasionados por mujeres son causados por el consumo de alcohol (OMS, 2014).

Según los datos de la Encuesta Nacional de Salud Mental (2015), el 17.8\% de los adolescentes, el $42.5 \%$ de los adultos entre 18 y 45 años, y el $27.2 \%$ de las personas mayores de 45 años han consumido alcohol en el último año, indicando además que el $21.8 \%$ de la muestra nacional de este estudio presentó un patrón de consumo excesivo de alcohol y el $11 \%$ un riesgo de abuso en el consumo (Ministerio de Salud y Protección Social, 2015). En este grupo etario, otros datos denotan bebedores de riesgo desde el $7.1 \%$ al $20.5 \%$ por cada región del país, y a nivel general del $9.1 \%$ en mujeres y del $16 \%$ en hombres (de la Espriella, Rodríguez, Rincón, Cabrera, Pérez, y Gómez-Restrepo, 2016). En estudiantes universitarios colombianos, las investigaciones muestran que el 93\% consume alcohol (Gantiva, Rodríguez, González, y Vera, 2011) y que entre el $38 \%$ y el $42 \%$ de ellos tienen un patrón de consumo perjudicial (Gantiva, Bello, Vanegas, y Sastoque, 2010; Montaño, Morales, Gómez, Vera, y Gantiva, 2011). Otro estudio (Cardona y Orjuela, 2014) en universitarios de Medellín indicó un 62\% con un consumo de riesgo, $31 \%$ con síntomas de dependencia y un $7 \%$ con consumo perjudicial, siendo mayor en hombres y en estudiantes de estrato alto.

Aunque los niveles y las consecuencias del consumo de alcohol son amplios, son pocos los instrumentos de tamizaje y diagnóstico validados que existen para este comportamiento. Uno de los instrumentos más utilizados a nivel mundial es el Cuestionario para Identificación de Trastornos Debidos al Consumo de Alcohol (AUDIT), el cual está constituido por 10 ítems de cuatro opciones de respuesta desarrollado para clasificar el consumo de alcohol en cuatro zonas (bajo riesgo o abstinentes, riesgo moderado, alto riesgo y posible dependencia) (Saunders, Aasland, Babor, De la Fuente, y Grant, 1993). El AUDIT ha sido validado por la OMS en diferentes países (Babor, Higgins, Saunders, y Monteiro, 2001) y en población universitaria, debido a los altos índices de consumo en esta población, por ejemplo, en estudiantes españoles (García, Novalbos, Martínez, y O'Ferrall, 2016), mexicanos (Gómez, Gómez, Morales, y Pérez, 2009) y chilenos (Palma, Manrique, y Barriga, 2013), y debe resaltarse como la escala con mayor uso en los estudios de trastorno por consumo de alcohol, a nivel de Latinoamérica (Cremonte et al., 2018), lo cual unido a la simpleza de la escala, su extensión, el soporte psicométrico internacional y la construcción desde la misma Organización mundial de la Salud, justifica su uso.

En población colombiana, un estudio realizado por Campo-Arias, Villamil-Vargas y Herazo (2013), con una muestra de 256 estudiantes de una facultad de medicina utilizando la versión en español del AUDIT, encontró que el instrumento presenta criterios de confiabilidad alta, sin embargo, la muestra es poco representativa de la población universitaria y no se llevó a cabo un proceso de validación y adaptación específico para esta población. Por lo anterior, el objetivo del presente estudio fue adaptar el Cuestionario para Identificación de Trastornos Debidos al Consumo de Alcohol (AUDIT) en universitarios colombianos, teniendo en cuenta la equivalencia del constructo, el idioma, la cultura, la validez de contenido, confiabilidad, consistencia interna, carga factorial y ajuste de los ítems.

\section{Método}

\section{Participantes}

En este proceso se hace una aplicación piloto a una muestra de 30 estudiantes, una aplicación a 557 univer- 
sitarios diferentes a los de la prueba piloto para el análisis factorial exploratorio, y completando la aplicación final con 1.557 estudiantes de 9 universidades diferentes de Bogotá, con edades comprendidas entre los 16 y 41 años $(M=21.26 ; D T=3.25)$, distribuidos en 820 hombres $(52.7 \%)$ y 737 mujeres $(47.3 \%)$, y obtenidos por medio de un muestreo aleatorio simple al interior de cada institución, esta muestra para el análisis factorial confirmatorio. La totalidad de aplicaciones se hicieron en el periodo de julio de 2016 y julio de 2017.

\section{Instrumentos}

Se utilizaron dos instrumentos; el primero es el formato de evaluación por jueces, que permite establecer la validez de contenido, teniendo en cuenta los criterios de redacción, pertinencia, estructura y lenguaje.

El segundo es el Cuestionario para Identificación de Trastornos Debidos al Consumo de Alcohol (AUDIT), diseñado por la Organización Mundial de la Salud (OMS) en 1982 publicado en 1989 y actualizado en 1992, fue desarrollado para detectar el consumo excesivo de alcohol, comprende 10 preguntas, donde los tres primeros ítems hacen referencia al consumo de riesgo, los tres siguientes exploran posibles síntomas de dependencia y los cuatro últimos evalúan el consumo perjudicial. Las preguntas de la 1 a la 8 puntúan entre 0 y 4 y las 9 y 10 puntúan 0,2 o 4 arrojando una puntuación máxima del instrumento de 40, y una mínima de cero, el AUDIT en su estudio original presenta la siguiente clasificación: puntuaciones iguales a cero reflejan abstinencia, puntuación entre uno y siete indican un consumo ocasional, puntuaciones entre 8 y 15 indican un posible consumo de riesgo, puntuaciones entre 16 y 19 indican un posible consumo perjudicial, y un consumo igual o mayor a 20 indican una posible dependencia al alcohol. Véase el Anexo I para una descripción completa del cuestionario.

El AUDIT es un cuestionario que cuenta con niveles altos de sensibilidad y especificidad, la cual se encuentra entre .80 y .90 aproximadamente, presenta correlaciones de .88 con otras pruebas similares como el MAST y el CAGE, y con índices de confiabilidad y consistencia interna de .86. (Babor et al., 2001; Palma et al., 2013).

\section{Procedimiento}

Dado el interés del estudio, se analizan los ítems del instrumento AUDIT, los cuales se establecen a partir de los procedimientos de adaptación de pruebas, propuestos por Hambleton y Kanjee (1995), desarrollado en las siguientes fases:
Fase 1. Inicialmente se seleccionó el cuestionario (AUDIT), que dadas sus características esta prueba fue creada para uso internacional por la OMS, a quien se le solicitó información, y se tomó como aval, lo que en el documento de la OMS plantea "Este documento puede, sin embargo, ser libremente revisado, resumido, reproducido y traducido, en parte o en su totalidad pero no para la venta o para su utilización con fines comerciales" (Babor et al., 2001). A continuación se trabajó con la equivalencia del constructo en el idioma y la cultura a la cual se va a adaptar la prueba (población universitaria), siguiendo con la consulta con expertos de la cultura a la que se va a adaptar la prueba para determinar si los constructos existen y si tiene la misma definición, evaluando la conveniencia de la adaptación de la prueba. Para ello se contó con la participación de tres traducciones oficiales, siendo dos traductores técnicos y un traductor profesional en psicología. Una vez se realizaron las traducciones por separado, para evitar sesgos, se reunieron a los traductores para discutir los resultados y unificar los ajustes en una sola versión.

Fase 2. Seguido se pasó al proceso de validez de contenido a partir de juicio de expertos ( 3 jueces conceptuales y 3 jueces psicométricos) para revisar el contenido y ajustar el instrumento.

Fase 3. Luego se realizó una aplicación piloto, donde a partir de las observaciones y los resultados obtenidos se hacen los ajustes correspondientes. Estas aplicaciones ser realizaron con un grupo de estudiantes de tres facultades diferentes a la Psicología, para garantizar la heterogeneidad, y se realizaron en un auditorio distinto al de sus clases.

Fase 4. En esta fase se realizó, una vez los ajustes de la prueba piloto, la aplicación a la muestra para el análisis factorial exploratorio (realizados con el software SPSS versión 24). Las aplicaciones se llevaron a cabo por los investigadores y por asistentes entrenados para tal fin. Se decide realizar un análisis factorial exploratorio ya que se desconoce la estructura factorial para la muestra a trabajar, sustentado además en la diferencia en la estructura obtenida en diversos estudios.

Fase 5. La última fase hace referencia a la aplicación final para obtener los datos, que se revisaron por medio del análisis factorial confirmatorio, concluyendo con la prueba adaptada para la población colombiana.

Cabe resaltar que este estudio inicia tras la aprobación de los grupos de investigación y el comité de ética de cada institución.

\section{Análisis estadístico}

Se examinó la validez de contenido mediante la revisión de los jueces con el coeficiente de razón de vali- 
dez de contenido (coeficiente de Lawshe), continuando con la distribución de las preguntas en los diferentes factores a través del análisis factorial exploratorio de los ítems, siguiendo con los estadísticos a nivel descriptivo de las puntuaciones arrojadas por la aplicación del cuestionario, los diferentes coeficientes de consistencia interna y confiabilidad encontrados desde la teoría clásica de los test, y finalmente el análisis factorial confirmatorio. En el análisis factorial exploratorio, la extracción de los factores se llevó a cabo mediante el método de mínimos cuadrados no ponderados, y la rotación de los factores a través de rotación oblicua (oblimin). En el análisis factorial confirmatorio se utilizó el método de estimación de máxima verosimilitud. Los análisis se efectuaron con los programas AMOS y SPSS.

\section{Resultados}

En la Tabla 1 se indican las puntuaciones medias en los ítems relacionadas con cada uno de los cuatro criterios. Como puede observarse, los ítems presentan en los cuatro criterios unos valores medios altos ya que las puntuaciones variaron entre 3.1 y 3.8 .

Tabla 1. Puntuaciones medias de calificación de los seis jueces calificando los cuatro criterios

\begin{tabular}{ccccc}
\hline Ítem & Redacción & Pertinencia & Estructura & Lenguaje \\
\hline 1 & 3.5 & 3.8 & 3.6 & 3.8 \\
2 & 3.5 & 3.8 & 3.3 & 3.8 \\
3 & 3.5 & 3.8 & 3.6 & 3.8 \\
4 & 3.8 & 3.8 & 3.8 & 3.8 \\
5 & 3.3 & 3.8 & 3.8 & 3.8 \\
6 & 3.5 & 3.8 & 3.1 & 3.8 \\
7 & 3.8 & 3.8 & 3.8 & 3.8 \\
8 & 3.8 & 3.8 & 3.8 & 3.8 \\
9 & 3.8 & 3.8 & 3.8 & 3.8 \\
10 & 3.8 & 3.8 & 3.8 & 3.8 \\
\hline
\end{tabular}

Complementando la evaluación por jueces se evidencia en la Tabla 2 el coeficiente de razón de validez de contenido, según el cual las puntuaciones de 1 significan que todos los jueces la consideran esencial, las puntuaciones de .75 indican que siete jueces lo consideran esencial, y las puntuaciones de 0.5 indica que seis lo consideran al ítem esencial en su criterio. De acuerdo con las puntuaciones de los jueces, los datos de dicha tabla indican que en general los ítems se consideran esenciales.
Tabla 2. Índice del coeficiente de razón de validez en los cuatro criterios

\begin{tabular}{ccccc}
\hline Item & Redacción & Pertinencia & Estructura & Lenguaje \\
\hline 1 & 0.75 & 1 & 0.50 & 1 \\
2 & 0.75 & 1 & 0.50 & 1 \\
3 & 0.75 & 1 & 0.75 & 1 \\
4 & 1 & 1 & 1 & 1 \\
5 & 0.50 & 1 & 1 & 1 \\
6 & 0.75 & 1 & 0.50 & 1 \\
7 & 1 & 1 & 1 & 1 \\
8 & 1 & 1 & 1 & 1 \\
9 & 1 & 1 & 1 & 1 \\
10 & 1 & 1 & 1 & 1 \\
\hline
\end{tabular}

Con el fin de determinar si el tamaño muestral es suficiente $(n=557)$ para realizar el análisis factorial exploratorio y tras los ajustes de datos atípicos por medio de la prueba de distancias extremas de Mahalanobis (conservando un $n=551$ ), se procedió a realizar las medidas de adecuación muestral de Kaiser-Meyer-Olkin y la prueba de esfericidad de Bartlett. Los datos obtenidos $(\mathrm{KMO}=$ .728 ; Bartlett $p<.001)$ refieren que el tamaño muestral es apropiado y que la matriz de correlaciones es adecuada para realizar el análisis factorial, el cual se realizó por medio de la extracción de mínimos cuadrados no ponderados con rotación con método Oblimin. La Tabla 3 muestra la extracción obtenida para cada reactivo, teniendo en cuenta que se espera una extracción mínima de .3 para conservar los ítems. Los datos de la tabla 3 indican que la extracción de cada reactivo es adecuada para conservar la totalidad de los ítems, a excepción de los reactivos 9 y 10, donde la extracción no fue suficiente, aunque se conservan para revisar la estructura factorial.

Tabla 3. Extracción obtenida para cada reactivo del AUDIT

\begin{tabular}{ccc}
\hline & Inicial & Extracción \\
\hline Item1 & 1.000 & 0.370 \\
Item2 & 1.000 & 0.560 \\
Item3 & 1.000 & 0.664 \\
Item4 & 1.000 & 0.315 \\
Item5 & 1.000 & 0.399 \\
Item6 & 1.000 & 0.430 \\
Item7 & 1.000 & 0.439 \\
Item8 & 1.000 & 0.316 \\
Item9 & 1.000 & 0.278 \\
Item10 & 1.000 & 0.282 \\
\hline
\end{tabular}


Al establecer los factores que constituyen el cuestionario, al igual que la varianza total explicada, se hallaron dos factores claramente constituidos, con un $38.54 \%$ de varianza total acumulada, con un primer factor que explica el $24.17 \%$ y un segundo factor con una carga del $14.37 \%$ de dicha varianza explicada. Cabe resaltar que el número de componentes fue determinado por medio de la regla de Kaiser de autovalor superior a uno, la congruencia teórica de los reactivos, el soporte empírico de estudios anteriores y el análisis paralelo de Horn.

Para verificar la agrupación de los reactivos en dichos factores, se muestra (Tabla 4) la matriz de componentes rotados, que muestra al reactivo 1, 2 y 3 agrupados en un factor independiente, los cuales hacen referencia al consumo de alcohol y a los reactivos 4 al 10 en el segundo componente, que hacen referencia a los síntomas y consecuencias del consumo.

Tabla 4. Matriz de componentes rotados del AUDIT

\begin{tabular}{ccc}
\hline Ítem & Componente 1 & Componente 2 \\
\hline Item1 & & 0.438 \\
Item2 & & 0.723 \\
Item3 & & 0.804 \\
Item4 & 0.447 & \\
Item5 & 0.632 & \\
Item6 & 0.655 & \\
Item7 & 0.643 & \\
Item8 & 0.560 & \\
Item9 & 0.401 & \\
Item10 & 0.527 & \\
\hline
\end{tabular}

El análisis factorial confirmatorio reflejó que el modelo estructural de dos factores obtenido a partir del análisis factorial exploratorio se ajustaba bien a los datos. Los índices de ajuste obtenidos fueron los siguientes: $\chi 2$ $(32)=92.92(\mathrm{p}<.001)$, goodness of fit index $(\mathrm{GFI})=$ .987 , Comparative fit index $(\mathrm{CFI})=.984$, y root mean square error of approximation (RMSEA $=.036$. Los valores de las estimaciones paramétricas del modelo se indican en la Figura 1. Aunque no se especifican, observamos que las estructuras unifactorial o trifactorial se ajustaban mucho peor a los datos que el modelo de dos factores.

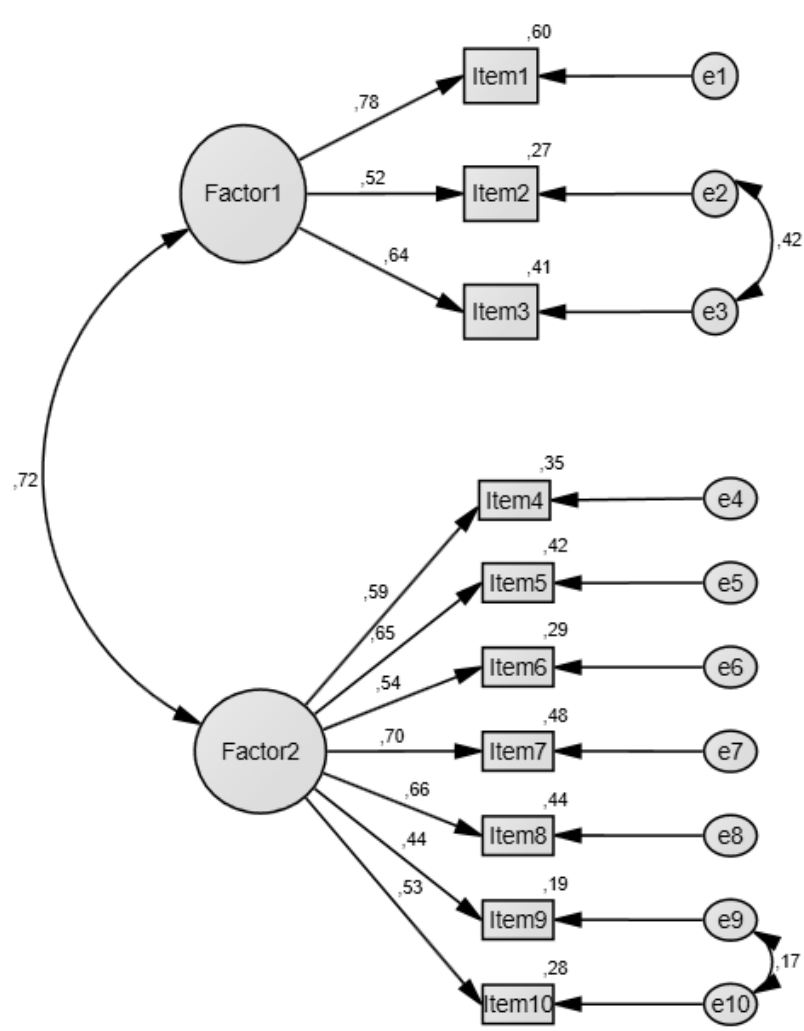

Figura 1. Modelo estructural del AUDIT.

Estos resultados indican que la estructura factorial del cuestionario es bastante robusta, demostrando que el AUDIT posee una estructura de dos factores suficientemente consistente (véase la Figura 1).

Por otro lado se realizaron análisis de fiabilidad, encontrando un alfa de Cronbach general del cuestionario de $.82, .73$ para el componente 1 y .79 para el segundo componente. Para determinar las correlaciones entre los elementos, como evidencia adicional de la consistencia interna de la escala, se muestran las matrices de correlaciones inter-elementos y las correlación elemento-total, tanto para la escala total, como para los dos componentes obtenidos, encontrando correlaciones positivas entre todos los reactivos, y correlaciones superiores a .30 entre todos los reactivos pertenecientes en sí a cada una de las subescalas, así como correlaciones superiores a .40 en todos los reactivos en comparación con la puntuación total de la escala y de cada subescala correspondiente (véanse las Tablas 5, 6 y 7). 
Tabla 5. Matriz de correlaciones inter-elementos y correlación elemento-total, para la escala total

\begin{tabular}{lcccccccccc}
\hline & Item2 & Item3 & Item4 & Item5 & Item6 & Item7 & Item8 & Item9 & Item10 & $\begin{array}{c}\text { Correlación elemento- } \\
\text { total corregida }\end{array}$ \\
\hline Item1 & .406 & .503 & .385 & .359 & .306 & .359 & .357 & .236 & .318 & .563 \\
Item2 & - & .579 & .284 & .210 & .164 & .219 & .266 & .399 & .199 & .440 \\
Item3 & & - & .388 & .347 & .299 & .327 & .340 & .262 & .297 & .605 \\
Item4 & & - & .405 & .355 & .397 & .397 & .316 & .339 & .551 \\
Item5 & & & - & .413 & .498 & .412 & .323 & .341 & .546 \\
Item6 & & & & - & .447 & .436 & .346 & .332 & .505 \\
Item7 & & & & & - & .496 & .315 & .428 & .573 \\
Item8 & & & & & & - & .355 & .372 & .573 \\
Item9 & & & & & & & - & .388 & .452 \\
Item10 & & & & & & & & - & .499 \\
\hline
\end{tabular}

Tabla 6. Matriz de correlaciones inter-elementos y correlación elemento-total, para el primer componente

\begin{tabular}{cccc}
\hline & Item2 & Item3 & $\begin{array}{c}\text { Correlación elemento- } \\
\text { total corregida }\end{array}$ \\
\hline Item1 & .406 & .503 & .501 \\
Item2 & - & .579 & .571 \\
Item3 & & - & .649 \\
\hline
\end{tabular}

Tabla 5. Matriz de correlaciones inter-elementos y correlación elemento-total, para la escala total

\begin{tabular}{cccccccc}
\hline & Item5 & Item6 & Item7 & Item8 & Item9 & Item10 & $\begin{array}{c}\text { Correlación elemento- } \\
\text { total corregida }\end{array}$ \\
\hline Item4 & .405 & .355 & .397 & .397 & .316 & .339 & .515 \\
Item5 & - & .413 & .498 & .412 & .323 & .341 & .560 \\
Item6 & & - & .447 & .436 & .346 & .332 & .545 \\
Item7 & & - & .496 & .315 & .428 & .608 \\
Item8 & & & - & .355 & .372 & .583 \\
Item9 & & & & - & .388 & .485 \\
Item10 & & & & & - & .528 \\
\hline
\end{tabular}

\section{Discusión}

Los resultados del presente estudio indican que el Cuestionario para Identificación de Trastornos Debidos al Consumo de Alcohol (AUDIT) está compuesta por 2 componentes claramente definidos, que explican el $38.54 \%$ de la varianza total acumulada, siendo conformado el primer factor por las preguntas 1 a la 3 y que hacen referencia al consumo de alcohol, mientras que las restantes siete preguntas refieren las consecuencias negativas experimentadas cuando se bebe. Cabe destacar que este modelo se confirmó a través de las ecuaciones estructurales, rectificándolo.

Los hallazgos encontrados sobre la estructura bifactorial difieren del encontrado por los autores originales de la validación de la OMS (Babor et al., 2001), pero concuerdan con los encontrados por estudios con jóvenes universitarios españoles (García et al., 2016) y chilenos 
(Palma et al., 2013) y el estudio exploratorio realizado con universitarios colombianos por Torres, Riveros, Torres y Gallego (2016). Es posible que la diferencia en la estructura esté dada por condiciones socioculturales del consumo, al comparar a universitarios norteamericanos e hispanohablantes, por lo cual se recomienda realizar estudios comparativos para afirmar esta postura explicativa.

Al respecto de los datos de consistencia y fiabilidad, se halló un coeficiente alfa de Cronbach de .82 para la escala total, .73 para el componente 1 y .79 para el segundo componente, así como correlaciones positivas entre todos los reactivos y superiores a 0.3 en los reactivos de cada subescala. Los datos de fiabilidad de esta escala se asemejan a los encontrados por Babor et al. (2001) en el estudio original, los da Palma et al. (2013) en Chile, los de García et al. (2016) y los de Torres et al. (2016) en Colombia, lo que rectifica la consistencia de la escala y su confiabilidad.

Sobre este estudio, sin embargo, hay algunas limitaciones. Aun cuando el tamaño muestral es amplio, la selección fue aleatoria y los estadísticos soportaron el número de participantes, cabe indicar que esta investigación se realizó con estudiantes vinculados a universidades de la ciudad de Bogotá, por lo que se sugiere replicar la presente investigación en otros contextos universitarios distintos, para verificar que dicha estructura hallada se mantenga, así como la replicación de esta investigación, no solo con universitarios sino con otras poblaciones. Por último, este estudio pretende mostrar la utilidad de la adaptación del AUDIT como herramienta de tamizaje para el consumo de alcohol, refiriendo que la misma tiene importantes evidencias de validez y confiabilidad, sugiriendo su uso en población universitaria, y recomendando estudios de prevalencia, comparación entre sexos, carreras y otras variables que permitan dar cuenta del consumo de alcohol y sus variables asociadas en universitarios colombianos.

\section{Referencias}

Babor, T., Higgins, J., Saunders, J., \& Monteiro, M. (2001). The Alcohol Use Disorders Identification Test Guidelines for Use in Primary Care, second edition. Recuperado el 16 de junio de 2016 de http://whqlibdoc.who.int/hq/2001/who_msd_msb_01.6a.pdf

Campo-Arias, A., Villamil-Vargas, M., y Herazo, E. (2013). Confiabilidad y dimensionalidad del AUDIT en estudiantes de medicina. Psicología desde el Caribe, 30(1), 21-35.

Cardona, J. y Orjuela, L. (2014). Consumo de riesgo, síntomas de dependencia y consumo perjudicial de alcohol en estudiantes de una universidad pública de Medellín-2013. Archivos de medicina, 10(1), 1-15.

Cremonte, M., Biscarra, M. A., Conde, K., \& Cherpitel, C. J. (2018). Epidemiology of alcohol consumption and related problems in Latin American countries: Contributions of psychology. International Journal of Psychology, 53(4), 245-252.

de la Espriella, R., Rodríguez, V., Rincón, C., Cabrera, D., Pérez, S., y Gómez-Restrepo, C. (2016). Consumo de alcohol en la población colombiana. Encuesta Nacional de Salud Mental 2015. Revista Colombiana de Psiquiatría, 45(1), 76-88.

Gantiva, C., Bello, J., Vanegas, E., y Sastoque, Y. (2010). Relación entre el consumo excesivo de alcohol y esquemas maladaptativos tempranos en estudiantes universitarios. Revista Colombiana de Psiquiatría, 39(2), 362-374.

Gantiva, C., Rodríguez, M., González, M., y Vera, A. (2011). Perfil de personalidad en consumidores moderados y excesivos de sustancias psicoactivas. Psicología desde el Caribe, 28(1), 24-38.

García, M., Novalbos, J., Martínez, J., y O’Ferrall, C. (2016). Validación del test para la identificación de trastornos por uso de alcohol en población universitaria: AUDIT y AUDIT-C. Adicciones, 28(4), 194-204.

Gómez. E., Gómez, H., Morales, B., y Pérez, M. (2009). Uso del AUDIT y el DAST-10 para la identificación de abuso de sustancias psicoactivas y alcohol en adolescentes. Revista Colombiana de Psicología, 18(1), 9-17.

Grant, B. F., Goldstein, R. B., Saha, T. D., Chou, S. P., Jung, J., Zhang, H., ... \& Hasin, D. S. (2015). Epidemiology of DSM-5 alcohol use disorder: results from the National Epidemiologic Survey on Alcohol and Related Conditions III. JAMA Psychiatry, 72(8), 757-766.

Hambleton, R. K. \& Kanjee, A. (1995). Increasing the Validity of Cross-Cultural Assessments: Use of Improved Methods for Test Adaptations. European Journal of Psychological Assessment, 11(3), 147-157.

Ministerio de Salud y Protección Social. (2015). Encuesta Nacional de Salud Mental 2015. Recuperado el 31 de mayo de 2016 de http://www.odc.gov.co/portals/1/ publicaciones/pdf/consumo/ estudios/nacionales/co031102015-salud_mental_tomoi.pdf

Montaño, M., Morales, A., Gómez, M., Vera, A., y Gantiva, C. (2015). Consumo de alcohol en estudiantes universitarios. Psicogente, 14(25), 27-35.

Motos, P., Cortés, M., Gimenez, J., y Cadaveira, F. (2015). Predictores del consumo semanal de alcohol y sus consecuencias asociadas en universitarios consumidores intensivos de alcohol. Adicciones, 27(2), 119-131.

Organización Mundial de la Salud. (2014). Global status report on alcohol and health 2014. Recuperado el 31 de mayo de 2016 de http://www.who.int/substance_abuse/publications/ global_alcohol_report/en/

Palma, F., Santander, G., y Barriga, O. (2013). Validez y confiabilidad del test de identificación de los trastornos debidos al consumo de alcohol (AUDIT), en estudiantes de universidad chilena. Ciencia y Enfermería, 19(1), 23-35.

Torres, L., Riveros, F., Torres, L., y Gallego, O. (2016). Validez y confiabilidad del cuestionario para identificación de trastornos debidos al consumo de alcohol (Audit) en universitarios colombianos. Revista Electrónica de Psicología Iztacala, 19(3), $1040-1052$.

Saunders, J., Aasland, O., Babor, T., De la Fuente, J. \& Grant, M. (1993). Development of the alcohol use disorders identification test (AUDIT): WHO collaborative project on early detection of persons with harmful alcohol consumption-II, Addiction, 88(6), 791-804. 


\section{Anexo I. Alcohol Use Disorders Identification Test (AUDIT)}

\section{PRUEBA DE IDENTIFICACIÓN DE TRASTORNOS POR CONSUMO DE ALCOHOL}

Debido a que el uso del alcohol puede afectar su salud e interferir con ciertos medicamentos y tratamientos, es importante que le hagamos algunas preguntas sobre su consumo de alcohol. Sus respuestas serán confidenciales, así que sea honesto por favor.

Marque con una $(\mathrm{X})$ el número que mejor represente su respuesta

1. ¿Con qué frecuencia, consume una bebida alcohólica?

(0) Nunca [Pase a las Preguntas 9-10]

(1) Una vez al mes o menos.

(2) 2 a 4 veces al mes.

(3) 2 a 3 veces a la semana.

(4) 4 o más veces a la semana.

2. ¿Cuántas bebidas alcohólicas consume normalmente en un día cuando bebe?

(0) 1 ó 2

(1) 3 ó 4

(2) 5 ó 6

(3) 7,8 , ó 9

(4) 10 ó más.

3. ¿Con qué frecuencia, consume seis o más bebidas alcohólicas en una misma ocasión?

(0) Nunca.

(1) Menos de una vez al mes.

(2) Mensualmente.

(3) Semanalmente.

(4) Diariamente o casi diariamente.

4. ¿Con qué frecuencia, durante el último año, le ha sucedido que no puede detenerse después de empezar a beber?

(0) Nunca.

(1) Menos de una vez al mes.

(2) Mensualmente.

(3) Semanalmente.

(4) Diariamente o casi diariamente

5. ¿Con qué frecuencia, durante el último año, ha dejado de hacer lo que normalmente se esperaba de usted, debido a la bebida?

(0) Nunca.

(1) Menos de una vez al mes.

(2) Mensualmente.

(3) Semanalmente.

(4) Diariamente o casi diariamente.
6. ¿Con qué frecuencia, durante el último año, ha necesitado un primer trago en la mañana para empezar el día, después de haber bebido mucho?

(0) Nunca.

(1) Menos de una vez al mes.

(2) Mensualmente.

(3) Semanalmente.

(4) Diariamente o casi diariamente.

7. ¿Con qué frecuencia, durante el último año, se ha sentido culpable o con remordimiento después de beber?

(0) Nunca.

(1) Menos de una vez al mes.

(2) Mensualmente.

(3) Semanalmente.

(4) Diariamente o casi diariamente.

8. ¿Con qué frecuencia, durante el último año, le ha sido imposible recordar lo que sucedió la noche anterior, por haber estado bebiendo?

(0) Nunca.

(1) Menos de una vez al mes.

(2) Mensualmente.

(3) Semanalmente.

(4) Diariamente o casi diariamente

9. ¿Usted, o alguien más, ha resultado lesionado como consecuencia de su consumo de bebidas alcohólicas?

(0) No.

(2) Sí, pero no en el último año.

(4) Sí, durante el último año.

10. ¿Algún familiar, amigo, médico u otro profesional de la salud ha manifestado preocupación por su nivel de consumo, o le han sugerido disminuirlo?

(0) No

(2) Sí, pero no en el último año.

(4) Sí, durante el último año. 